\title{
EVALUACIÓN DEL BIOESTIMULANTE FOLIAR (BIOAGRO TRIPLE A) EN LA PRODUCCIÓN DE TOMATE TIPO CHONTO (LYCOPERSICUM SCULENTUM MILL) EN DOS AMBIENTES DE CULTIVO
}

EVALUATION OF THE FOLIAR BIOSTIMULANT (BIOAGRO TRIPLE A) IN THE PRODUCTION OF CHONTO TOMATO (LYCOPERSICUM SCULENTUM MILL) IN TWO GROWING ENVIRONMENTS

\section{Diego Alejandro Agudelo $\mathrm{T}$. \\ Universidad Nacional Abierta y a Distancia}

\author{
Manuel Francisco Polanco Puerta \\ Ingeniero Agrónomo. Esp. MSc. PhD. \\ Docente - Universidad Nacional Abierta y a Distancia UNAD \\ manuel.polanco@unad.edu.co
}

\section{RESUMEN.}

El tomate, es el cultivo hortícola de consumo en fresco más importante en el mundo y en Colombia. En el departamento de Risaralda se cultivan alrededor de 3.000 hectáreas bajo cubiertas plásticas, sin un paquete tecnológico adecuado que permita lograr la mayor rentabilidad del cultivo. En los últimos años, se ha implementado la aplicación de productos foliares que complementan la fertilización edáfica, como es el caso de los bioestimulantes o fertilizantes foliares con acción bioestimulante, convirtiéndose en una práctica común entre los cultivadores de tomate. La presente investigación tuvo como objeto evaluar el efecto del producto bioestimulante Bioagro Triple A, sin registro ante el ICA, producto que pretende ser una alternativa de fertilización foliar complementaria de bajo costo para una producción sostenible del cultivo de tomate tipo chonto. La evaluación se realizó, en las instalaciones de la Universidad Nacional Abierta y a Distancia UNAD, ubicada en el Municipio de Dosquebradas Risaralda a $1.450 \mathrm{msnm}$ y con precipitación promedio de $2.700 \mathrm{~mm}$

DOI: https://doi.org/10.22490/26653176.3423 
anuales, en dos ambientes: Bajo invernadero y a campo abierto. Se estableció un diseño experimental en bloques completos al azar, con tres tratamientos y 10 repeticiones. Las variables evaluadas fueron: altura de la planta, diámetro del tallo, numero de hojas, índice de área foliar, numero de racimos florales, numero de flores, numero de frutos, peso de frutos y producción total por tratamiento. Los resultados mostraron que el producto Bioagro Triple A puede ser una excelente alternativa económica y sostenible para los productores de este cultivo.

anuales, en dos ambientes: Bajo invernadero y a campo abierto. Se estableció un diseño experimental en bloques completos al azar, con tres tratamientos y 10 repeticiones. Las variables evaluadas fueron: altura de la planta, diámetro del tallo, numero de hojas, índice de área foliar, numero de racimos florales, numero de flores, numero de frutos, peso de frutos y producción total por tratamiento. Los resultados mostraron que el producto Bioagro Triple A puede ser una excelente alternativa económica y sostenible para los productores de este cultivo.

Palabras Clave: Fitohormonas; fertilizante; Foliar; Hortaliza; Invernadero

\section{ABSTRACT.}

Tomato, vegetable crop is the largest consumer in the world fresh and Colombia. In the department of Risaralda about 3,000 hectares are grown under plastic covers, without adequate technology package that will ensure greater profitability of the crop. In recent years, we have implemented the application of foliar products that complement the soil fertilization, as in the case of foliar fertilizers bioestimulantes or bioestimulante action, becoming a common practice among tomato growers. The present study was to evaluate the effect of product bioestimulante BIOAGRO TRIPLE A, without registration with the ICA, product intended to be an alternative complementary low-cost foliar fertilization for sustainable production of tomato crop chonto type. The evaluation was conducted at the premises of the National Open University and Distance UNAD, located in the municipality of Dosquebradas Risaralda at 1,450 meters above sea level and average annual rainfall of $2,700 \mathrm{~mm}$ 
in two environments: Under greenhouse and open field. An experimental design was established in completely randomized, with three treatments and 10 repetitions blocks. The variables evaluated were plant height, stem diameter, number of leaves, leaf area index, number of flower clusters, number of flowers, number of fruits, fruit weight and total production per treatment. The results showed that the product BIOAGRO TRIPLE A can be an excellent economic and sustainable alternative for producers of this crop.

Keywords: Fitohormonas; fertilization; Foliar; Vegetable; Greenhouse.

\section{INTRODUCCIÓN.}

El tomate es originario de América del sur, entre las regiones de Chile, Ecuador y Colombia, pero su domesticación se inició en el sur de México y norte de Guatemala. Las formas silvestres de "tomate cereza", Lycopersicon esculentum var. cerasiforme, originarias de Perú, migraron a través del Ecuador, Colombia, Panamá y América Central hasta llegar a México, donde fue domesticado por el hombre; en la lengua nahua de México era llamado tomatl, que sin lugar a dudas dio origen a su nombre actual. (Jaramillo, Rodríguez, Guzmán, Zapata, \& Rengifo, 2007).

El tomate alcanzó un alto nivel de domesticación en México, antes de ser llevado a Europa y Asia. Los diferentes herbarios en Europa muestran descripciones y grabados de tomate solamente a partir de la segunda mitad del siglo XVI. De acuerdo con lo anterior, esto indica que los primeros cultivares en el continente europeo tenían frutos blandos, con amplia variedad de formas y colores, cambios que fueron realizados por los agricultores primitivos de México. (Jaramillo, et al., 2007).

La mayor extensión dedicada a este cultivo se encuentra en China, país en el que un total de 1'005 millones de hectáreas se dedican a producir tomate, con un volumen de 50'12 millones de toneladas. El segundo lugar por número de hectáreas lo ocupa India, donde a este cultivo se dedican 870.000 hectáreas de las que obtienen $17^{\prime} 5$ millones de toneladas. La 


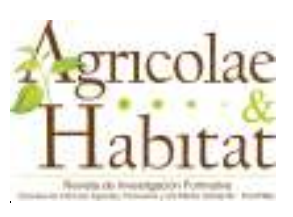

tercera posición por la extensión dedicada a cultivar tomate es para Turquía, con 300.000 hectáreas y una producción de 11'35 millones de toneladas. (ver figura 1). La cuarta posición es para Nigeria con 270.000 hectáreas, seguida por Egipto (216.395), Irán (160.000), Estados Unidos (150.140), Camerún (150.000), Rusia (117.700), apareciendo México en el décimo lugar por extensión dedicada al cultivo del tomate, con un total de 96.651 hectáreas. (Agriculturers, 2014)

En Colombia existe un área cultivada de tomate que oscila alrededor de las 17.250 ha, con un rendimiento en $\mathrm{Kg} / \mathrm{ha}$ de 39.213; cultivándose en 24 de los 32 departamentos, siendo Norte de Santander, Santander, Antioquia, Cundinamarca, Huila, Valle del Cauca y Boyacá los mayores productores con el $73 \%$ de la producción nacional. La producción del país a nivel mundial se encuentra alrededor de las 700.000 toneladas ocupando el lugar No 34 en cuanto a producción. El departamento más productor de tomate es Boyacá. (Ministerio de Agricultura y Desarrollo Rural, 2014).

La planta de tomate es anual, de porte arbustivo; se desarrolla de forma rastrera, semierecta o erecta, dependiendo de la variedad. Según el hábito de crecimiento, las variedades de tomate se clasifican en dos grupos: las variedades de crecimiento determinado, cuyos tallos terminan en un ramillete floral que marca el punto donde se termina el crecimiento, y las variedades de crecimiento indeterminado, que son aquellas de hábito guiador, cuyo ápice ubicado en la parte extrema del tallo, sigue creciendo indefinidamente. (Instituto de Investigaciones Agropecuarias, 2017)

El tomate es una planta autógama, es decir que su polinización se produce internamente con polen de la propia flor. La polinización cruzada puede existir y a veces puede superar el $5 \%$, lo cual puede ser un aspecto importante para mantener la pureza varietal. El desarrollo de yemas, flores, y frutos ocurre progresivamente dentro del racimo; en una planta, una flor abre antes que otra ya que es muy ocasional encontrar dos flores de un racimo en el mismo estado de desarrollo. La polinización autógama o normal se produce generalmente entre un 95 y 100\%. La polinización en el campo es producida por el viento que mueve las flores y eventualmente por insectos que con el vibrar de sus alas mueven la flor 
promoviendo la dehiscencia de las anteras; en invernaderos la función del viento e insectos es reemplazada por ventiladores o de forma manual moviendo los soportes donde se apoyan las plantas. (Argerich \& Gaviola, 1988).

El tomate tiene la capacidad de crecer en condiciones climáticas muy variadas, donde es habitual observar cultivares en zonas de altura y en zonas bajas o cálidas. El factor limitante de la producción en zonas altas es el riesgo de heladas o temperaturas bajas, condiciones que pueden afectar el desarrollo del cultivo. Las variedades de tomate que se siembran en zonas bajas o cálidas pueden verse perjudicados por la presencia de insectos plaga los cuales son limitantes también en la producción; en lo relacionado a enfermedades, éstas serán más o menos serias dependiendo de las condiciones de temperatura y humedad relativa de cada lugar. (CATIE, 1990).

Para minimizar de alguna manera los riesgos de tipo biótico y/o abiótico, los productores de tomate han optado por los invernaderos para asegurar la producción y calidad de las plantaciones, de una manera adecuada a lo largo de todo el año. El concepto de cultivos bajo invernadero representa el paso de producción extensiva de tomate a producción intensiva, suministrando las condiciones óptimas a las plantas desde la raíz a las hojas (Corpoica, 2006).

El manejo de la fertilización foliar y utilización de bioestimulantes en la agricultura es cada vez más frecuente por la demanda nutricional de los cultivos de alto rendimiento, donde el objetivo generalmente es suplir los requerimientos nutricionales en épocas críticas (caso micronutrientes esenciales); acortar o retardar ciclos en la planta e inducir etapas específicas fenológicas, además, de contrarrestar condiciones de stress en la planta; aporte energético en etapas productivas o nutrición foliar con fines de sanidad vegetal. (Gómez \& Castro, 2010 Hidalgo, et al. 1998).

El uso de bioestimulantes vía foliar, se refiere a la aplicación externa de sustancias en baja concentración generalmente menor al 0,25\% bien sea para activar o retardar procesos fisiológicos específicos principalmente en 
el crecimiento (raíz, ápices foliares, yemas) o para contrarrestar demandas energéticas o activación puntual de procesos en el desarrollo y sostenimiento de estructuras, además pueden en ocasiones incentivar la absorción de nutrientes como es el caso de algunos aminoácidos o ácidos carboxílicos de cadena corta o media; por otro lado se ha buscado incentivar procesos de defensa natural contra patógenos como es el caso de sustancias con base en fosfonatos, ácido salicílico, boratos. (Hernández, 2006; Microfertisa, 2012.)

Derivado del conocimiento de las hormonas naturales o sustancias inductoras producidas por las plantas y sus efectos sobre el desarrollo y productividad de las mismas, han surgido en el mercado un sin número de bioestimulantes (productos sintéticos y/o complejos que emulan a dichas hormonas químicas en su funcionamiento), así como algunos extractos de origen vegetal y marino que contienen algunas de esas hormonas naturales y los cuales son empleados en aplicaciones exógenas, con fines de lograr alguna ventaja comercial o competitiva. Los grupos de compuestos hormonales descubiertos y reportados hasta el momento y que tienen un impacto significativo sobre el desarrollo y manejo en los cultivos son los siguientes: Auxinas, Giberelinas, Citocininas, Etileno, Ácido Abscísico, Brasinoesteroides, Salicilatos, Jasmonatos. (Gómez \& Castro 2010, Cuellar, 2013, Cuesta, y Mondaca, 2014).

Es importante indicar, que el uso indiscriminado de productos químicos en la agricultura ha provocado un desequilibrio en la naturaleza, donde se han eliminado o desplazado organismos que ejercen un control biológico en los cultivos, situación que va en detrimento sobre la calidad biológica de los alimentos y sobre la calidad de vida del hombre, ya que son altamente derrochadores de energía y alteran completamente las propiedades físicas, químicas y biológicas del suelo. (Terry, Leyva, Ruiz, \& Díaz, 2009, Collantes Quintero, Castellanos, Peláez, \& Tamaris, 2014). Por tal razón es importante indicar, que, dentro de los paquetes de manejo de cultivo, se deben priorizar prácticas culturales con las cuales se garantice un equilibrio con el medio ambiente. 
Según Beltran, 2000, Las fitohormonas son moléculas orgánicas que en pequeñas cantidades pueden influir en el comportamiento y desarrollo de las plantas y éstas juegan un papel importante en el crecimiento, la floración, producción y la maduración de los cultivos. La aplicación de bioestimulantes del crecimiento vegetal con el objetivo de incrementar la calidad de las cosechas y sus rendimientos, es un aspecto dentro de las investigaciones agrícolas de gran importancia para la agricultura por las implicaciones de carácter social y económico que aporta. Sin embargo, es importante tener en cuenta que la aplicación de bioestimulantes vía foliar, debe llevarse a cabo de forma cuidadosa y ésta debe ajustarse a un plan de manejo de cultivo recomendado por profesionales del campo agrícola, con el fin de no alterar la fisiología de las plantas.

La aplicación de bioestimulantes a los cultivos va teniendo cada vez más importancia desde el punto de vista agronómico, porque actúan como estimuladores o reguladores del crecimiento de las plantas, lo que desde el punto de vista económico es favorable para los productores ya que se induce a los cultivos a que se aumenten las producciones. Los reguladores de crecimiento son aplicados en pequeñas cantidades y pueden aumentar, inhibir o modificar diferentes procesos fisiológicos de las plantas como la inducción de la germinación de semillas, el crecimiento, la acumulación de materia seca, la inducción floral, el llenado y maduración del fruto, entre otras, lo que permite mejorar y estandarizar las producciones. (Serrani, 2008; Cruz, et al. 2010).

Durante la realización del ensayo, se evaluó el efecto del producto bioestimulante, denominado "Bioagro Triple A" sobre las siguientes variables morfoagronómicas; Altura de la Planta; Diámetro del Tallo; Número Hojas; Índice de Área Foliar; Número Inflorescencias; Número Flores; Número Frutos; Peso Fruto y Producción Total por Planta, en tomate tipo chonto para el consumo en fresco (Lycopersicum sculentum Mill), con el fin de cuantificar la respuesta fisiológica de las plantas de tomate frente a la aplicación del producto Bioagro Triple A, en dos ambientes de cultivo. 
Los resultados de la investigación permitieron evidenciar que la aplicación foliar de bioestimulantes puede ser una herramienta que permite manejar el cultivo de tomate de una forma eficiente, siendo una alternativa técnica y económicamente viable al alcance de los productores que permite lograr altas producciones por cosecha, lo que redunda en un mayor ingreso y por lo tanto en la mejora de la calidad de vida de los agricultores que se dedican a esta línea productiva.

\section{METODOLOGÍA.}

Para el desarrollo de esta investigación, se utilizó un invernadero inteligente con ambiente controlado y con sistema de fertirrigación automático, controlado mediante sistema AUTOAGRONOM. Dentro del invernadero se mantuvieron los siguientes valores de variables climáticas:

Temperatura diurna promedio: 32 oC Temperatura nocturna promedio: 19 oC. Diferencia de temperatura día- noche: 13 oC; Humedad relativa: $85 \%$

En condiciones de campo abierto, se tuvo el mismo sistema automatizado de fertirrigación que dentro del invernadero y se registraron las siguientes variables climáticas:

Temperatura diurna promedio: 28 oC. Temperatura nocturna promedio: 18 oC. Diferencia de temperatura día- noche: 10 oC. Humedad relativa: $80 \%$

\section{Material de Siembra y Plantulación}

Se utilizó semilla de tomate tipo Chonto Híbrido Matador® de la compañía de semillas EUROSEMILLAS. Este material fue sembrado en bandejas plásticas de 200 alveolos dentro del invernadero sobre una cama de germinación con temperatura constante de $30^{\circ} \mathrm{C}$ y riego por microasperción cada 10 minutos. A los 20 días después de siembra se tuvieron plántulas con 2 hojas verdaderas las cuales fueron trasplantadas a las parcelas experimentales. 


\section{Adecuación de Camas y Parcelas}

Dentro del invernadero se usaron 9 camas de $17 \mathrm{mts}$ de largo $\times 1 \mathrm{mts}$ de ancho $\times 20 \mathrm{cms}$ de profundidad; en campo abierto se utilizaron 9 camas de $17 \mathrm{mts}$ de largo $\times 1 \mathrm{mts}$ de ancho $\times 20 \mathrm{cms}$ de profundidad; antes de llevar las plántulas al sitio definitivo, se realizó la adecuación de las eras o del terreno, donde se llevaron a cabo las siguientes actividades: desyerba, picado de terreno para el buen desarrollo radicular de las plantas, aplicación de Trichoderma al suelo a razón de $3 \mathrm{gr} / \mathrm{l}$ de agua; como sustrato de siembra se utilizó en la plantulación turba y en las eras suelo normal. Finalmente se cubrió el suelo con plástico negro (Agromulch) en cada era para el control de arvenses.

\section{Establecimiento del Ensayo}

En cada cama se independizaron parcelas de $17 \mathrm{~m} 2$, en donde se establecieron al azar los tratamientos, en cada parcela se ubicaron 40 plantas. Las plántulas fueron trasplantadas a los 20 días después de la siembra, cuando éstas contaban con dos pares de hojas verdaderas. La siembra de las plantas de tomate se realizó a una distancia de $30 \mathrm{~cm}$ entre plantas y $1,5 \mathrm{~m}$ entre surcos, para una densidad de siembra de 2,22 plantas por metro cuadrado o 22.222 por hectárea.

\section{Productos bioestimulantes aplicados}

Se realizaron aplicaciones de los productos Bioagro Triple A (sin registro) y Tecnoverde $®$.

Bioagro Triple A: Composición a base de Citocininas, Giberelinas, Auxinas, Vitaminas, Boro y Coadyuvantes (es un producto en experimentación).

Tecno Verde®: Composición a base los siguientes aminoácidos libres: ácido aspártico, ácido glutámico, serina, glicina, histidina, arginina, treonina, alanina, prolina, tisonina, valina, metionina, cisteína, isoleucina, leucina, fenilalanina, lisina.

La aplicación foliar de los bio estimulantes se hizo de la siguiente manera: 
Primera Aplicación: se efectuó una primera aplicación a las plántulas en las bandejas, antes de ser trasplantadas a su sitio definitivo; se aplicó el producto a razón de $1,5 \mathrm{cc} /$ /t de agua y de esta solución se aplicó 1,5 litros a la bandeja, con el propósito de que esta aplicación inicial de la formulación penetrara en la zona radicular de las plantitas.

Segunda Aplicación: Se hizo a los 20 días después del transplante de las plántulas de tomate a una dosis de $1.5 \mathrm{cc}$ por litro de agua asperjándose la solución en toda la planta.

Tercera Aplicación: Se hizo a los 20 días después de hacer la segunda aplicación

Cuarta Aplicación y Subsiguientes: Estas se realizaron 20 días después de cada aplicación para un total de 7 aplicaciones.

\section{Manejo de Cultivo}

El invernadero y el lote a campo abierto cuentan con sistema de fertirrigación, por lo tanto, no se utilizaron fertilizantes por este medio; solo se aplicó por este medio agua a las plantas para mantenerlas hidratadas. Se realizaron 7 aplicaciones de 20 gramos del fertilizante

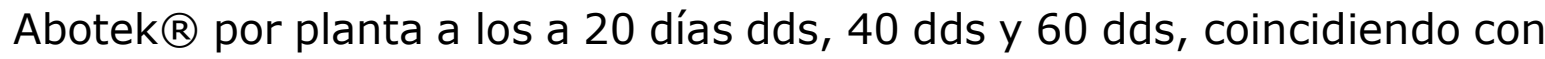
las épocas de aplicación de bioestimulantes por vía foliar.

\section{Diseño experimental}

Se empleó un diseño completamente al azar, con tres tratamientos $\times 10$ repeticiones, en 2 dos ambientes de cultivo (invernadero y campo abierto). Las plantas fueron sembradas en eras a una distancia de $30 \mathrm{~cm}$ entre plantas, para lo cual se sembraron 40 plantas de tomate por tratamiento. La variedad de tomate utilizada en el experimento fue Chonto Híbrido Matador.

Tratamiento 1: Aplicación de Bioagro Triple A en dosis de $1.5 \mathrm{ml}$ por litro de agua.

Tratamiento 2: Aplicación de Tecnoverde $®$ en dosis de 1,5 ml por litro de agua. 
Tratamiento 3: Testigo absoluto sin aplicación de bioestimulantes.

La unidad experimental correspondió a las parcelas, con un área de 153 m2 y población de 360 plantas. La unidad de muestreo fue una planta completa por tratamiento, por repetición y por muestreo.

\section{DISCUSIÓN Y RESULTADOS}

Evaluación del comportamiento morfoagronómico del tomate en dos ambientes

En la Tabla 1 se muestran los resultados obtenidos de la prueba DUNCAN, la cual determina las diferencias entre las variables de respuesta, según los tratamientos al cual fue sometido el cultivo del tomate en los dos ambientes de cultivo y el análisis de todas las interacciones, evidenciándose que: las variables Altura de la Planta (AP) e Índice Área Foliar (IAF) presentan los mejores resultados dentro del invernadero; esta situación también pudo presentarse no solo por el efecto en la aplicación de los bioestimulantes, si no también a la menor luz solar directa, que ocasionaron un alargamiento de la planta; en cuanto al Diámetro del Tallo (DT), el mejor resultado se presentó en el ambiente campo abierto; frente a la variable Número de Hojas $(\mathrm{NH})$, no se presentaron diferencias significativas en los 2 ambientes evaluados, donde se concluye que ni los productos ni el ambiente afectaron la emisión de hojas

En las variables Número de Inflorescencias (NI) y Número de Flores (NF) en el ambiente invernadero, presentaron diferencias estadísticas significativas; sin embargo en las variables Número de Frutos (NFr), Peso del Fruto (PFr) y Producción Total por Planta (PTp), los mejores resultados se presentan en el ambiente de cultivo campo abierto, queriendo decir esto que hubo un mejor comportamiento de los productos Bioestimulantes en este ambiente de cultivo y que estos estimularon la formación, llenado y peso del fruto. En algunos momentos por pequeñas fallas en los equipos que controlan la temperatura en el invernadero provocaron que se presentaran altas temperaturas (mayores a $30 \mathrm{oC}$ ) lo que pudo haber favorecido el aborto floral y la caída de flores; por tal 
razón los mejores resultados en las variables NFr, PFr y PTp fueron campo abierto donde hubo menos caída de flores y buen cuajamiento.

Tabla 1 Prueba Duncan análisis por sitio combinado para cada variable evaluada

\begin{tabular}{|c|c|c|c|c|c|}
\hline \multirow{3}{*}{$\begin{array}{l}\text { SITIO } \\
\text { Invernadero } \\
\text { Campo } \\
\text { Abierto }\end{array}$} & AP & DT & \multicolumn{2}{|r|}{ NH } & IAF \\
\hline & $125,22 \mathrm{~A}$ & $0,84 \mathrm{~B}$ & \multicolumn{2}{|r|}{$13,59 \mathrm{~A}$} & $305,72 \mathrm{~A}$ \\
\hline & $99,15 \mathrm{~B}$ & $0,97 \mathrm{~A}$ & \multicolumn{2}{|r|}{$13,65 \mathrm{~A}$} & $231,38 \mathrm{~B}$ \\
\hline \multicolumn{6}{|l|}{ Continuación } \\
\hline SITIO & NI & NF & NFr & PFr & PTp \\
\hline Invernadero & $2,39 \mathrm{~A}$ & $4,22 \mathrm{~A}$ & $7,14 \mathrm{~B}$ & $98,35 \mathrm{~B}$ & $579,14 \mathrm{~B}$ \\
\hline $\begin{array}{l}\text { Campo } \\
\text { Abierto }\end{array}$ & $2,21 \mathrm{~B}$ & $3,33 \mathrm{~B}$ & $10,22 \mathrm{~A}$ & $138,51 \mathrm{~A}$ & $1574,50 \mathrm{~A}$ \\
\hline
\end{tabular}

De acuerdo con la Tabla 2, en la variable Altura de la Planta (AP) el mejor resultado se presentó con el tratamiento realizado con el producto Tecnoverde; en cuanto al Diámetro del Tallo (DT), los tratamientos realizados con Bioagro Triple A y Tecnoverde, no presentaron diferencias estadísticas; en el descriptor Número de Hojas, el mejor resultado los presentó el producto Tecnoverde frente a los otros 2 tratamientos utilizados (Bioagro Triple A y el Testigo absoluto); en cuanto al Índice del Área Floral los tratamientos Bioagro Triple A y Tecnoverde no presentaron diferencias estadísticas significativas pero si existen diferencias significativas frente al testigo.

Así mismo, se presentan diferencias estadísticas significativas en las variables Número de Frutos (NFr) y Producción Total por Planta (PTp) en el tratamiento con Bioagro Triple $A$. Para las variables Número de Inflorescencias (NI), Número de Flores (NF) y Peso del Fruto (PFr) no se presentaron diferencias estadísticas en los tratamientos realizados con Bioagro Triple A y Tecnoverde. 
Tabla 2. Prueba Duncan análisis tratamiento combinado para cada variable evaluada

\begin{tabular}{|c|c|c|c|c|c|}
\hline TRATAMIENTO & AP & \multicolumn{2}{|l|}{ DT } & NH & IAF \\
\hline Bioagro AAA & $110,82 \mathrm{~B}$ & \multicolumn{2}{|c|}{$0,92 \mathrm{~A}$} & $13,40 \mathrm{~B}$ & $271,42 \mathrm{~A}$ \\
\hline Tecnoverde & $115,33 \mathrm{~A}$ & \multicolumn{2}{|l|}{$0,91 \mathrm{~A}$} & $14,37 \mathrm{~A}$ & $281,51 \mathrm{~A}$ \\
\hline Testigo & $110,25 \mathrm{~B}$ & \multicolumn{2}{|l|}{$0,87 \mathrm{~B}$} & $13,10 \mathrm{~B}$ & $252,73 \mathrm{~B}$ \\
\hline \multicolumn{6}{|l|}{ Continuación } \\
\hline TRATAMIENTO & NI & NF & NFr & PFr & PTp \\
\hline Bioagro AAA & $2,33 \mathrm{~A}$ & $3,81 \mathrm{AB}$ & $9,33 \mathrm{~A}$ & $126,42 \mathrm{~A}$ & $1292,45 \mathrm{~A}$ \\
\hline Tecnoverde & $2,40 \mathrm{~A}$ & $3,97 \mathrm{~A}$ & $8,66 \mathrm{~B}$ & $126,78 \mathrm{~A}$ & $1107,33 \mathrm{~B}$ \\
\hline Testigo & $2,17 \mathrm{~B}$ & $3,57 \mathrm{~B}$ & $8,07 \mathrm{~B}$ & $102,09 \mathrm{~B}$ & $830,69 \mathrm{C}$ \\
\hline
\end{tabular}

Valores entre columnas seguidas por letras diferentes son estadísticamente diferentes $(p<0,05)$ de acuerdo con la prueba Duncan.

De acuerdo con los resultados que se presentan en la Tabla 3 la variable Número de Frutos (NFr), no presenta diferencias estadísticas significativas en los 3 tratamientos utilizados, queriendo decir esto que los productos no causaron efecto en este descriptor cuantitativo; sin embargo en las variables Peso del Fruto (PFr) y Producción Total por Planta (PTp) aunque en los tratamientos realizados con Bioagro Triple A y Tecnoverde no presentan diferencias estadísticas significativas, si se aprecia que existen diferencias frente al testigo absoluto por lo cual se concluye que la aplicación de los productos Bioestimulantes si son importantes para la formación, llenado y peso del fruto.

Tabla 3. Prueba Duncan análisis por tratamiento para cada variable evaluada

\begin{tabular}{lccc}
\hline TRATAMIENTO & NFr & PFr & PTp \\
Bioagro AAA & $84,95 \mathrm{~A}$ & $760,70 \mathrm{~A}$ & $7754,70 \mathrm{~A}$ \\
Tecnoverde & $80,60 \mathrm{~A}$ & $758,55 \mathrm{~A}$ & $6644,00 \mathrm{~A}$ \\
Testigo & $75,95 \mathrm{~A}$ & $612,55 \mathrm{~B}$ & $4984,20 \mathrm{~B}$ \\
$\begin{array}{l}\text { Valores entre columnas seguidas por letras diferentes son estadísticamente diferentes }(p<0,05) \\
\text { de acuerdo con la prueba Duncan. }\end{array}$
\end{tabular}




\section{CONCLUSIONES.}

El producto Bioagro Triple A presento un buen comportamiento de respuesta en la producción de tomate, al ser aplicado como un bioestimulante en ambos ambientes de cultivo, comparado con el producto Tecnoverde.

Las plantas que fueron tratadas con el producto Bioagro Triple A presentaron efectos altamente significativos en las variables número de frutos formados, queriendo decir esto que el producto evita el aborto de frutos, favorece a que haya un mayor cuajamiento y finalmente a que se obtenga una mayor producción.

Los resultados obtenidos en las plantas tratadas con Bioagro Triple A en las variables Número de Frutos, Peso de Fruto y Producción Total por Planta fueron mayores a campo abierto que los obtenidos bajo invernadero. Esto se debe a que en algunos momentos la temperatura del invernadero sobrepaso los $30^{\circ} \mathrm{C}$ y esto ocasionó caída de flores lo que repercute directamente en la producción; así mismo el efecto de falta de luminosidad por el plástico del invernadero que es de aspecto lechoso, pudo afectar la baja producción ya que la planta requiere energía para desarrollar sus procesos fisiológicos.

Los caracteres morfoagronómicos del cultivo de tomate de mesa, que son afectados favorablemente con la aplicación de productos bioestimulantes tanto a campo abierto como dentro del invernadero son el número y el peso de los frutos.

De acuerdo con los resultados obtenidos en el experimento, el producto Bioagro Triple A en fase de experimentación, podría ser una alternativa importante y económica de manejo de cultivo de tomate para los productores ya que es un producto que induce floración, formación, llenado, peso y calidad del fruto.

\section{BIBLIOGRAFÍA.}

Agriculturers. (9 de Octubre de 2014). Red de especialistas en agricultura. Recuperado el 9 de Marzo de 2016, de http://agriculturers.com/el- 
tomate-ocupa-en-el-mundo-casi-cinco-millones-de-hectareas/: http://agriculturers.com

Argerich, C., \& Gaviola, J. (1988). Manual de producción de semillas hortícolas. Buenos Aires, Buenos Aires, Argentina.

Beltran, A. L. (2000). Aplicación de Hormonas y Fertilizantes Foliares en el Cultivo de Tomatillo ( Physalis ixocarpa Brot.). Saltillo, Coahuila, México.

CATIE. (1990). Guía para el manejo integrado de plagas del cultivo de tomate. Informe técnico No 151, Centro Agronómico Tropical de Investigación y Enseñaza.

Collantes Quintero, A., Castellanos-Barliza, J., León Peláez, J., \& TamarisTurizo, C. (2014). Caracterización de materia orgánica aportada por hojarasca fina en los bosques de ribera del río Gaira (Sierra Nevada de Santa Marta - Colombia). Revista de Investigación Agraria y Ambiental, 5(1), 171-184. doi:https://doi.org/10.22490/21456453.946

Corpoica. (2013). Tecnología para el Cultivo del Tomate Bajo Condiciones Protegidas.

Cruz, M., Melgarejo, L. M., \& Romero, M. (2010). Fitohormonas. Bogotá, Cundinamarca, Colombia.

Cuellar, A. (2013). Respuesta de la Aplicación de Algas Marinas en la Producción de Tomate (Lycopersicon esculentum Mill) Convencional y Orgánico Bajo Invernadero. Torreón, Coahuila, México.

Cuesta, G., \& Mondaca, E. (2014). Efecto de un biorregulador a base de auxinas sobre el crecimiento de plantines de tomate. Revista Chapingo. Serie Horticultura, 1-8.

DANE. (2014). El cultivo de tomate de mesa bajo invernadero tecnología que ofrece mayor producción, calidad e inocuidad del producto. Boletín 
mensual insumos y factores asociados a la producción agropecuaria (30), 72.

Gómez, M., \& Castro , H. (2010). Manejo de la fertilización foliar y bioestimulantes. Recuperado el 20 de Febrero de 2016, de Infojardín: http://www.infojardin.com

Hernández , P. (2006). Aplicación de un aminoácido experimental en la germinación de plántulas de tomate (Lycopersicon esculentum Mill var. florarade) bajo invernadero. Saltillo, Coahuila, México.

Hidalgo, J. C., Alcántar, G., Baca, G., Sánchez, P., \& Escalante, A. (1998). Efecto de la condicion nutrimental de las plantas y de la composicion, concentracion y ph del fertilizante foliar, sobre el rendimiento y calidad en tomate. Red de Revistas Científicas de América Latina y el Caribe, España y Portugal , 16 (2), 143-148.

Instituto de Investigaciones Agropecuarias. (2017). Manual de cultivo del tomate al aire libre. Santiago, Chile.

Jaramillo, J., Rodríguez , V., Guzmán, M., Zapata, M., \& Rengifo, T. (2007). Manual Técnico Buenas Prácticas Agrícolas -BPA- en la Producción de Tomate Bajo Condiciones Protegidas (Primera edición ed.). Medellín, Antioquia, Colombia: CTP Print LTDA.

Jordán, M., \& Casaretto, J. (2006). Hormonas y reguladores del crecimiento: auxinas, giberelinas y citocininas. La Serena, La Serena, Chile.

Leiva, S. (2010). Efectos de cuatro bioestimulantes sobre el crecimiento y peso en plántulas de almácigos de tomate (Lycopersicon esculentum). Costa Rica.

Microfertisa. (2012 de Enero de 2012). La Fertilización foliar, una herramienta que complementa la nutrición de sus cultivos. Recuperado el 15 de Octubre de 2015, de Microfertisa: http://www.microfertisa.com.co 
Ministerio de Agricultura y Desarrollo Rural. (2014). Producción de tomate en Colombia.

Pimienta , A. (2004). Ácidos Húmicos y Fúlvicos de Origen Orgánico en el Crecimiento de Plántula de Tomate (Lycopersicon esculentum Mill.) en Invernadero. Saltillo, Coahuila, México.

Ramírez, E., Castillo, C., Aceves, E., \& Carrillo , E. (2004). EFECTO DE PRODUCTOS CON REGULADORES DE CRECIMIENTO SOBRE LA FLORACIÓN Y AMARRE DE FRUTO EN CHILE 'HABANERO. Chapingo serie Horticultura, 1 (11), 93-98.

Restrepo, E., Vallejo, F., \& Lobo, M. (2008). Fenología de la floración en tomate cultivado y especies silvestres relacionadas. Acta Agronómica, 57 (2), 89-93.

Reyes , A., Albarrán, J., Benavides, A., López, R., Alonso, R., \& Rodríguez Evangelina. Efecto de los amonoácidos en el crecimiento y producción del tomate. Colombia.

Salamanca, M., \& Alvarado, A. (2012). Efecto de la proteína harpin y el fosfito de potasio en el control de mildeo polvoso (Frysiphe polygoni D.C.) en tomate, en Sutamarchán (Boyacá) (Vol. 9). Colombia.

Serrani, J. (2008). Interacción de Giberelinas y Auxinas en la Fructificación del Tomate. Valencia, España.

Terry , E., Leyva, A., Ruiz, J., \& Díaz, M. M. (2009). Biostan. Un producto natural con efectividad biológica en el cultivo del tomate (Solanum lycopersicon L.). CENIC. Ciencias Biológicas, 40 (2), 89-92.

Vuelta-Lorenzo, D. R., Vidal-Cuevas, Y., Rizo-Mustelier, M., Bell-Mesa, T., \& Molina-Lores, L. B. (2017). EFECTO DEL BRASINOESTEROIDE FOLIAR (BIOBRAS 16) SOBRE EL CRECIMIENTO Y LA PRODUCCIÓN DEL CULTIVO DE FRIJOL (PHASEOLUS VULGARIS, L.). Ciencia en su PC, (3), 1-12. 
Agnicolae

Habitat 